\title{
ON THE REGULARITY INDEX OF $n+3$ ALMOST EQUIMULTIPLE FAT POINTS IN $\mathbb{P}^{n}$
}

\author{
Nguyen Chanh TU and Tran Manh HUNG \\ (Received 4 April 2012 and revised 23 October 2012)
}

\begin{abstract}
The aim of this paper is to prove a well-known conjecture on the regularity index of $n+3$ almost equimultiple fat points in $\mathbb{P}^{n}$.
\end{abstract}

\section{Introduction}

We denote by $\mathbb{P}^{n}$ the projective space over an algebraically closed field $k$. Let $P \in \mathbb{P}^{n}$; then we say that a form $f$ of the polynomial ring $R:=k\left[x_{0}, \ldots, x_{n}\right]$ has multiplicity $m$ at $P$ if all partial derivatives of $f$ of order $<m$ vanish at $P$.

Let $X:=\left\{P_{1}, \ldots, P_{s}\right\} \subset \mathbb{P}^{n}$, let $m_{1}, \ldots, m_{s}$ be positive integers, and denote by $Z:=$ $m_{1} P_{1}+\cdots+m_{s} P_{s}$ the zero-scheme corresponding to the ideal of all forms of $R$ vanishing at $P_{i}$ with multiplicity at least $m_{i}$, for $i=1, \ldots, s$. This zero-scheme is called a set of fat points.

Let $\wp_{1}, \ldots, \wp_{s}$ denote the defining ideals of $P_{1}, \ldots, P_{s}$, respectively. Put

$$
I:=R / \wp_{1}^{m_{1}} \cap \cdots \cap \wp_{s}^{m_{s}} .
$$

Since the coordinate ring $R / I$ of the zero-scheme $Z$ is a one-dimensional Cohen-Macaulay graded $k$-algebra, the Hilbert function $H_{R / I}(t):=\operatorname{dim}_{k}(R / I)_{t}$ is strictly increasing until it reaches the multiplicity

$$
e(R / I)=\sum_{i=1}^{s}\left(\begin{array}{c}
m_{i}+n-1 \\
n
\end{array}\right)
$$

at which it stabilizes. Note that the Hilbert polynomial $P_{R / I}(t)$ of $R / I$ is constant and equal to $e(R / I)$. The least integer $t$ for which $H_{R / I}(t)=P_{R / I}(t)=e(R / I)$ is called the regularity index of $Z$ and is denoted by $\operatorname{reg}(Z)$. It is well known that $\operatorname{reg}(Z)$ is equal to the Castelnuovo regularity index reg $(R / I)$ (see [9] for a definition of the Castelnuovo regularity index of any $k$-algebra).

It was proved in [5] that $\operatorname{reg}(Z)=\left(\sum_{i=1}^{s} m_{i}\right)-1$ when the points are all on a line. Except for special cases, computation of $\operatorname{reg}(Z)$ is difficult. Then one tries to find (possibly sharp) upper bounds for it.

Such a (sharp) upper bound was given by B. Segre in 1961 for fat points in general position in $\mathbb{P}^{2}$ (see $[2, \mathbf{1 0}]$ ) and was extended to fat points in general position in $\mathbb{P}^{n}$ by 2010 Mathematics Subject Classification: Primary 13P05, 13H10; Secondary 14Q99, 13H15, $13 \mathrm{D} 40$.

Keywords: regularity index; zero-scheme; Hilbert function; fat points.

(C) 2013 Faculty of Mathematics, Kyushu University 
Catalisano et al in 1993 (see [4]). The bound was given by

$$
\max \left\{\max _{i, j}\left(m_{i}+m_{j}-1\right),\left[\frac{1}{n} \sum_{i=1}^{s} m_{i}+n-2\right]\right\}
$$

and it is called the Segre bound.

The Segre bound was extended to any set of fat points in $\mathbb{P}^{2}$ by Fatabbi in 1994 (see [6]) and, with different methods, by Thien in 1999 (see [11]).

In 1996, N. V. Trung formulated the following conjecture (see [12]) which was independently given by G. Fatabbi and A. Lorenzini (see [7]).

Conjecture. Let $Z:=m_{1} P_{1}+\cdots+m_{s} P_{s}$ be a set of fat points in $\mathbb{P}^{n}$. For $j=1, \ldots, n$, let

$$
T_{j}:=\max \left\{\left[\frac{1}{j}\left(m_{i_{1}}+m_{i_{2}}+\cdots+m_{i_{q}}+j-2\right)\right] \mid P_{i_{1}}, P_{i_{2}}, \ldots, P_{i_{q}} \text { lie on a } j \text {-plane }\right\},
$$

then

$$
\operatorname{reg}(Z) \leq T_{Z}:=\max \left\{T_{j} \mid j=1, \ldots, n\right\} .
$$

The upper bound $T_{Z}$ is an extended form of the Segre bound and therefore it is called the general Segre bound. The conjecture was proved for the case of fat points in $\mathbb{P}^{3}$ by Thien in 2000 (see [12]) and independently by Fatabbi and Lorenzini in 2001 (see [7]). Thien also proved in 2002 the case of double points in $\mathbb{P}^{4}$ (see [13]). Recently, Benedetti et al [1] have proved the case of non-degenerate $n+2$ fat points in $\mathbb{P}^{n}$. Note that, for any set $Z$ of $s<n+2$ fat points not in general position in $\mathbb{P}^{n}$, there exists an $(s-2)$-plane containing $Z$. It means that the result for the case of $n+2$ fat points in $\mathbb{P}^{n}$ implies that the conjecture holds for all cases of smaller numbers of fat points in $\mathbb{P}^{n}$.

A set of fat points is called almost equimultiple if the multiplicities of the points are equal to $k$ or $k-1$ for a given integer $k \geq 2$. In this paper, we use the algebraic method as indicated in [14] to prove the conjecture for any set of $n+3$ almost equimultiple, non-degenerate fat points in $\mathbb{P}^{n}$.

In the next section, we show several results which will be used to prove the conjecture in Section 3, and explain why the case of $n+3$ fat points is more complicated than the case of $n+2$ fat points.

\section{Auxiliary results}

The algebraic method used to estimate $\operatorname{reg}(Z)=\operatorname{reg}(R / I)$ in this paper as well as in $[\mathbf{1}, \mathbf{4}$, 11-13] is based on the following lemma.

Lemma 2.1. [4, Lemma 1] Let $P_{1}, \ldots, P_{r}, P$ be distinct points in $\mathbb{P}^{n}$ and let $\wp$ be the defining prime ideal of $P$. If $m_{1}, \ldots, m_{r}$, a are positive integers, $J:=\wp_{1}^{m_{1}} \cap \cdots \cap \wp_{r}^{m_{r}}$, and $I:=J \cap \wp^{a}$, then

$$
\operatorname{reg}(R / I)=\max \left\{a-1, \operatorname{reg}(R / J), \operatorname{reg}\left(R /\left(J+\wp^{a}\right)\right)\right\} .
$$


In the above lemma, the Castelnuovo regularity index $\operatorname{reg}\left(R /\left(J+\wp^{a}\right)\right)$ of $k$-algebra $R /\left(J+\wp^{a}\right)$ is equal to the least integer $t$ such that $\left(R /\left(J+\wp^{a}\right)\right)_{t}=0$.

In order to use the lemma, we choose a relevant point $P_{i_{0}}$ in the support of $Z$ and let $J:=\bigcap_{j \neq i_{0}} \wp_{j}^{m_{j}}$. Since reg $(R / J)$, in many cases, can be computed by induction on the number of fat points, one needs to estimate $\operatorname{reg}\left(R /\left(J+\wp_{i_{0}}^{m_{i_{0}}}\right)\right)$, for which the following lemma provides a useful tool.

Lemma 2.2. [4, Lemma 3] Let $P_{1}, \ldots, P_{r}, P$ be distinct points in $\mathbb{P}^{n}$ and let $\wp$ be the defining prime ideal of $P$. If $m_{1}, \ldots, m_{r}$, a are positive integers, $J:=\wp_{1}^{m_{1}} \cap \cdots \cap \wp_{r}^{m_{r}}$ and $\wp=\left(x_{1}, \ldots, x_{n}\right)$, then $\operatorname{reg}\left(R /\left(J+\wp^{a}\right)\right) \leq b$ if and only if $x_{0}^{b-i} M \in J+\wp^{i+1}$ for any monomial $M \in \wp^{i}$, where $i=0, \ldots, a-1$.

In practice, we choose a relevant number $T$, then for any monomial $M \in \wp^{i}$ where $0 \leq i \leq a-1$, we show that there exist hyperplanes $H_{1}, \ldots, H_{T-i}$ avoiding $P$ and satisfying $H_{1} \cdots H_{T-i} M \in J$. We can write $H_{j}=L_{j}+x_{0}$, where $L_{j} \in \wp$ for $j=1, \ldots, T-i$. Then

$$
H_{1} \cdots H_{T-i} M=\left(L_{1}+x_{0}\right) \cdots\left(L_{T-i}+x_{0}\right) M \in J
$$

implies $x_{0}^{T-i} M \in J+\wp^{i+1}$. This means that $\operatorname{reg}\left(R /\left(J+\wp^{a}\right)\right) \leq T$.

In particular, we have the following corollary.

Corollary 2.3. Let $Z:=m_{1} P_{1}+\cdots+m_{s} P_{s}$ be a set of fat points in $\mathbb{P}^{n}$. Let $J:=$ $\wp_{1}^{m_{1}} \cap \cdots \cap \wp_{s-1}^{m_{s-1}}$ and suppose that $P_{1}, \ldots, P_{s-1}$ are contained in a hyperplane avoiding $P_{s}$. Then $\operatorname{reg}\left(R /\left(J+\wp_{s}^{m_{s}}\right)\right) \leq T_{Z}$.

The result of the above corollary is attained by noting that if $H$ is a hyperplane containing $P_{1}, \ldots, P_{s-1}$ and avoiding $P_{s}$, then $H^{T_{Z}-i} \in J$ since $T_{Z}-i \geq T_{Z}-m_{s}+1 \geq m_{j}$ for all $0 \leq i \leq m_{s}-1$ and $1 \leq j \leq s-1$.

For the case $s=n+2$ and supposing that the points are not in general position and nondegenerate, then there exists a hyperplane $H$ containing $n+1$ points and not containing the remain one, say $P_{i_{0}}$. Therefore in this case, the corollary shows that $\operatorname{reg}\left(R / J+\wp_{i_{0}}^{m_{i_{0}}}\right) \leq T_{Z}$ immediately.

For the case $s=n+3$ fat points and supposing that they are non-degenerate and not in general position, it could be possible that there is no hyperplane containing $s-1=n+2$ points of the support of the given set of fat points. Therefore showing the existence of $P_{i_{0}}$ or proving $\operatorname{reg}\left(R / J+\wp_{i_{0}}^{m_{i_{0}}}\right) \leq T_{Z}$ is more complicated even for the case of equimultiple fat points.

Moreover, for the case that there exists a hyperplane $H$ containing $s-1=n+2$ points and avoiding the remain point $P_{i_{0}}$, then by Lemma 2.1, we still need to show that $\operatorname{reg}(R / J) \leq T_{Z}$, where $J:=\bigcap_{j \neq i_{0}} \wp_{j}^{m_{j}}$. Note that the ideal $J$ corresponds to the set of fat points $Y$ whose support lies on a hyperplane $H \cong \mathbb{P}^{n-1}$. Denote by $Y^{\prime}$ the set of fat points in $\mathbb{P}^{n-1}$ corresponding to $Y$, then by induction we have $\operatorname{reg}\left(Y^{\prime}\right) \leq T_{Y^{\prime}}$. The following lemma shows that, in fact, $T_{Y^{\prime}}$ is still an upper bound of $\operatorname{reg}(Y)=\operatorname{reg}(R / J)$.

LeMma 2.4. [1, Lemma 4.4] Let $Y:=m_{1} P_{1}+\cdots+m_{s-1} P_{s-1}$ be a set of fat points in $\mathbb{P}^{n}$ whose support is contained in a hyperplane $H \cong \mathbb{P}^{n-1}$. Denote by $P_{j}^{\prime}$ the point of $\mathbb{P}^{n-1}$ corresponding to $P_{j}$, for $1 \leq j \leq s-1$. Let $Y^{\prime}:=m_{1} P_{1}^{\prime}+\cdots+m_{s-1} P_{s-1}^{\prime}$ be the set of fat points in $\mathbb{P}^{n-1}$ corresponding to $Y$. If there is a non-negative integer $t$ such that $\operatorname{reg}\left(Y^{\prime}\right) \leq t$ then $\operatorname{reg}(Y) \leq t$. 


\section{Main results}

In this section, we show that the conjecture holds for any set of $n+3$ almost equimultiple fat points in $\mathbb{P}^{n}$. We only consider the cases of $n+3$ fat points which are non-degenerate in $\mathbb{P}^{n}$ to avoid the fact that all the points lie on a hyperplane. Due to the result in [4], we only need to consider the case that the support $X=\left\{P_{1}, \ldots, P_{n+3}\right\}$ of a set of fat points $Z$ is not in general position. Set

$$
d:=\min \{t \mid \exists t \text {-plane containing } t+2 \text { points of } X\} .
$$

Then it is easy to see that $1 \leq d \leq n-1$.

As mentioned in the previous section, our method is based on Lemma 2.1. Therefore, in each case, we firstly choose $P_{i_{0}}$ in the support $X$ of $Z$, put $J:=\bigcap_{j \neq i_{0}} \wp_{j}^{m_{j}}$ and show that $\operatorname{reg}\left(R / J+\wp_{i_{0}}^{m_{i_{0}}}\right) \leq T_{Z}$.

We divide the section into several parts. In the first subsection, we show a definition of coverings of a set of fat points and elementary properties in order to have a convenient method for studying $\operatorname{reg}\left(R / J+\wp_{i_{0}}^{m_{i}}\right)$. In the second subsection, we show covering properties of a set of $n+2$ almost equimultiple fat points. These properties give upper bounds of $\operatorname{reg}\left(R / J+\wp_{i_{0}}^{m_{i_{0}}}\right)$ according to sets of $n+3$ almost equimultiple fat points in $\mathbb{P}^{n}$. The last section is intended to check the conjecture for this case.

\subsection{Coverings of a set of fat points}

Definition. Let $s, a$ be non-negative integers and let $a \geq 1$. A fat point $Z:=m_{1} P_{1}+$ $\cdots+m_{r} P_{r}$ is called $(s, a)$-covered if for every collection $\mathcal{C}$ of $i$ hyperplanes of the form $x_{j}=0, j=1, \ldots, n$ (not necessary distinct), $i=0, \ldots, a-1$, there exists a collection $\mathcal{D}$ of $s-i$ hyperplanes avoiding $P=e_{0}:=(1,0, \ldots, 0)$ such that there are at least $m_{t}$ hyperplanes in $\mathcal{C} \cup \mathcal{D}$ containing $P_{t}$ for any $t=1 \ldots, r$.

A trivial verification shows the following properties.

Lemma 3.1. If $m_{1} P_{1}+\cdots+m_{r} P_{r}$ is $(s, a)$-covered, then $n_{1} P_{1}+\cdots+n_{r} P_{r}$ is $(q, b)$ covered for any sequence of non-negative integers $n_{t} \leq m_{t}, t=1, \ldots, r$, and for any $q \geq s$, $1 \leq b \leq a$.

LEMMA 3.2. If $m_{1} P_{1}+\cdots+m_{r} P_{r}$ is $(s, a)$-covered and $n_{1} P_{1}+\cdots+n_{r} P_{r}$ is $(t, b)$ covered, then $\left(m_{1}+n_{1}\right) P_{1}+\cdots+\left(m_{r}+n_{r}\right) P_{r}$ is $(s+t, a+b-1)$-covered.

In particular, with $n_{1}=\cdots=n_{r}=0, b=t+1$, we have the following lemma.

LEMMA 3.3. If $m_{1} P_{1}+\cdots+m_{r} P_{r}$ is $(s, a)$-covered, then it is $(s+t, a+t)$-covered for any non-negative integer $t$.

The meaning of the definition is shown in the following lemma.

LeMmA 3.4. Let $\wp_{1}, \ldots, \wp_{r}, \wp$ be the ideals of $P_{1}, \ldots, P_{r}, P$, respectively, let $J:=$ $\bigcap_{i=1}^{r} \wp_{i}^{m_{i}}$ and $P=e_{0}$. If $m_{1} P_{1}+\cdots+m_{r} P_{r}$ is $(s, a)$-covered, then $\operatorname{reg}\left(R /\left(J+\wp^{a}\right)\right) \leq s$.

In the next subsection, we will study covering properties of a set of $n+2$ almost equimultiple fat points in $\mathbb{P}^{n}$. 


\subsection{Covering properties of a set of $n+2$ almost equimultiple fat points}

Proposition 3.5. Let $X:=\left\{P_{1}, \ldots, P_{n+3}\right\}$ be a non-degenerate set of distinct points, not in general position in $\mathbb{P}^{n}, n \geq 4$, and there are no $n+2$ points of $X$ lying on a hyperplane. Let $d$ be the smallest number such that there exists a d-plane containing $d+2$ points of $X$. Assume that $P_{1}, \ldots, P_{d}, P_{n+2}, P_{n+3}$ lie on a d-plane and $P_{n+3}=e_{0}$. Then:

(1) $P_{1}+2 P_{2}+\cdots+2 P_{n-1}+P_{n}+P_{n+1}+P_{n+2}$ is $(2,1)$-covered. Consequently, $P_{1}+$ $P_{2}+\cdots+P_{n+2}$ is $(2,1)$-covered and hence $2 P_{1}+2 P_{2}+\cdots+2 P_{n+2}$ is $(4,1)$ covered;

(2) $P_{1}+P_{2}+\cdots+P_{n+2}$ is $(2,2)$-covered if $d \geq 2$;

(3) $2 P_{1}+\cdots+2 P_{n+2}$ is $(3,1)$-covered if $d \geq 2$;

(4) $2 P_{1}+\cdots+2 P_{n+2}$ is $(3,2)$-covered if $d \geq 3, n \geq 5$.

Proof. We can assume that $P_{i}=e_{i}$, where $e_{1}=(0,1,0, \ldots, 0), \ldots, e_{n}=(0, \ldots, 0,1)$.

(1) Let $H_{1}=V\left(x_{0}\right)$ and $H_{2}$ be the hyperplane determined by $P_{2}, \ldots, P_{n-1}, P_{n+1}, P_{n+2}$ then $P_{i} \in H_{1}, i=1, \ldots, n$, and $P_{n+3} \notin H_{1} \cup H_{2}$. It is clear that

$$
P_{1}+2 P_{2}+\cdots+2 P_{n-1}+P_{n}+P_{n+1}+P_{n+2}
$$

is $(2,1)$-covered.

(2) According to the definition and from case (1), we now need only to check for $i=1$. Consider $K=V\left(x_{j}\right)$.

If $1 \leq j \leq d$, then the plane containing $P_{j}, P_{n+1}, P_{n+2}$ does not contain $P_{n+3}$ since $d \geq 2$. Choose $H$ to be a hyperplane containing $P_{j}, P_{n+1}, P_{n+2}$ and avoiding $P_{n+3}$ then $P_{i} \in K \cup H, i=1, \ldots, n+2$. If $K=V\left(x_{j}\right)$ and $d<j \leq n$, then $P_{n+2}, P_{i} \in K, 1 \leq i \neq$ $j \leq n$. Since there are no 3 points of $X$ collinear, let $H$ be a hyperplane containing $P_{j}, P_{n+1}$ and avoiding $P_{n+3}$, then we have $P_{i} \in K \cup H, i=1, \ldots, n+2$. This means that $P_{1}+P_{2}+\cdots+P_{n+1}$ is $(2,2)$-covered if $d \geq 2$.

(3) Since $d \geq 2$, we see that the 3-plane determined by $P_{n}, P_{n+1}, P_{n+2}, P_{n+3}$ does not contain at least one point in $\left\{P_{1}, \ldots, P_{d}\right\}$, which we can assume to be $P_{1}$. This means that $P_{1}+P_{n}+P_{n+1}+P_{n+2}$ is $(1,1)$-covered. Moreover $P_{1}+2 P_{2}+\cdots+2 P_{n-1}+P_{n}+$ $P_{n+1}+P_{n+2}$ is $(2,1)$-covered, by Lemma 3.2, we have $2 P_{1}+\cdots+2 P_{n+2}$ is $(3,1)$-covered if $d \geq 2$.

(4) Since $2 P_{1}+\cdots+2 P_{n+2}$ is $(3,1)$-covered, according to the covering definition, we only need to check for $i=1$. Consider $K=V\left(x_{j}\right)$, then $K$ contains $P_{i}, 1 \leq i \neq j \leq n$. The proof is divided into 2 cases.

Case 1: $1 \leq j \leq d$. Since $d \geq 3$, there exist distinct integers $t, u$ such that $1 \leq t \neq i \neq$ $u \leq d$. One of the points in $\left\{P_{t}, P_{u}\right\}$, say $P_{t}$, is not contained in the 4-plane determined by $P_{j}, P_{n}, P_{n+1}, P_{n+2}, P_{n+3}$. Let $H_{1}$ be a hyperplane containing the 4-plane determined by $P_{t}, P_{j}, P_{n}, P_{n+1}, P_{n+2}$ and avoiding $P_{n+3}$. Let $H_{2}$ be the hyperplane determined by $P_{n+1}, P_{n+2}, P_{i}, 1 \leq i \neq t \leq n-1$, then $P_{n+3} \notin H_{2}$. It is clear that there exist two of $\left\{K, H_{1}, H_{2}\right\}$ containing $P_{i}$ for any $1 \leq i \leq n+2$.

Case 2: $d<j \leq n$. In this case the hyperplane $K=V\left(x_{j}\right)$ contains $P_{n+2}, P_{i}, 1 \leq i \neq j \leq n$.

If $d \leq n-2$, then there exists an integer $s$ such that $d<j \neq s \leq n$. There exists $1 \leq$ $t \leq d$ such that $P_{t}$ is not contained in the 3-plane determined by $P_{j}, P_{s}, P_{n+1}, P_{n+3}$. Let $H_{1}$ 
be a hyperplane containing $P_{t}, P_{j}, P_{s}, P_{n+1}$ and avoiding $P_{n+3}$. Let $H_{2}$ be the hyperplane determined by all $P_{i}, i \notin\{t, s\}, i \leq n+2$, then $P_{n+3} \notin H_{2}$. It is clear that there exist two of $\left\{K, H_{1}, H_{2}\right\}$ containing $P_{i}$ for any $1 \leq i \leq n+2$.

If $d=n-1$ then $j=n$ and the hyperplane $K=V\left(x_{n}\right)$ contains $P_{n+2}, P_{i}, 1 \leq$ $i \leq n-1$. One of $P_{1}, P_{2}$, say $P_{2}$, is not contained in the hyperplane determined by $P_{3}, \ldots, P_{n+1}, P_{n+3}$. Let $H_{1}$ be the hyperplane determined by $P_{2}, \ldots, P_{n+1}$, then $P_{n+3} \notin$ $H_{1}$. Since $d=n-1 \geq 4$, the 3 -plane determined by $P_{1}, P_{n}, P_{n+1}, P_{n+2}$ does not contain $P_{n+3}$. Let $H_{2}$ be a hyperplane containing $P_{1}, P_{n}, P_{n+1}, P_{n+2}$ and avoiding $P_{n+3}$. It is clear that there exist two of $\left\{K, H_{1}, H_{2}\right\}$ containing $P_{i}$ for any $1 \leq i \leq n+2$.

This means that $2 P_{1}+\cdots+2 P_{n+2}$ is $(3,2)$-covered and the proof is complete.

Corollary 3.6. With the hypothesis as in Proposition 3.5, we have the following:

(1) $m_{1} P_{1}+2 P_{2}+\cdots+2 P_{n+1}+m_{n+2} P_{n+2}$ is $(3,1)$-covered, where $m_{1}, m_{n+2} \geq 1$, $m_{1}+m_{n+2}=3$;

(2) $2 P_{1}+3 P_{2}+\cdots+3 P_{n+1}+2 P_{n+2}$ is $(5,2)$-covered;

(3) for $n=4, d=3,2 P_{1}+2 P_{2}+2 P_{3}+P_{4}+2 P_{5}+2 P_{6}$ is (3,2)-covered and $3 P_{1}+$ $\cdots+3 P_{6}$ is $(5,3)$-covered.

Proof. Choose coordinates such that $P_{i}=e_{i}, i=1, \ldots, n$.

(1) By Proposition 3.5, we have $P_{1}+2 P_{2}+\cdots+2 P_{n-1}+P_{n}+P_{n+1}+P_{n+2}$ is (2, 1)covered. On the other hand, both $P_{1}+P_{n}+P_{n+1}$ and $P_{n}+P_{n+1}+P_{n+2}$ are $(1,1)$-covered, the result is attained by Lemma 3.2.

(2) If $d \geq 2$, we know from Proposition 3.5 that $P_{1}+\cdots+P_{n+2}$ is $(2,2)$-covered and $P_{1}+$ $2 P_{2}+\cdots+2 P_{n+1}+P_{n+2}$ is $(3,1)$-covered as in the above. Then the assertion follows by Lemma 3.2. If $d=1$, then $P_{1}+2 P_{2}+\cdots+2 P_{n-1}+P_{n}+P_{n+1}+P_{n+2}$ is $(2,1)$-covered. Similarly, it is easily seen that $P_{1}+P_{2}+P_{3}+\cdots+2 P_{n}+2 P_{n+1}+P_{n+2}$ is $(2,1)$-covered. This implies that $2 P_{1}+3 P_{2}+\cdots+3 P_{n+1}+2 P_{n+2}$ is $(4,1)$-covered and hence $(5,2)$ covered.

(3) We see that $P_{1}+2 P_{2}+2 P_{3}+P_{4}+P_{5}+P_{6}$ is $(2,1)$-covered by Proposition $3.5, P_{1}+$ $P_{5}+P_{6}$ is $(1,1)$-covered. Thus $2 P_{1}+2 P_{2}+2 P_{3}+P_{4}+2 P_{5}+2 P_{6}$ is $(3,1)$-covered.

Consider $K=V\left(x_{1}\right)$, we see that $P_{2}, P_{3}, P_{4} \in K$. Consider hyperplane $H_{1}$ determined by $P_{1}, P_{2}, P_{5}, P_{6}$ and hyperplane $H_{2}$ determined by $P_{1}, P_{3}, P_{5}, P_{6}$, then $P_{7} \notin H_{1} \cup H_{2}$. Note that $P_{4} \in K$ and there exist two of $\left\{K, H_{1}, H_{2}\right\}$ containing $P_{i}$ for any $1 \leq i \leq 6, i \neq 4$. A similar consideration applies to the case $K=V\left(x_{j}\right), j=2,3$.

If $K=V\left(x_{4}\right)$, then $P_{i} \in K, i=1,2,3$. Any of two planes determined by $\left\{P_{1}, P_{2}, P_{5}\right\}$ and $\left\{P_{1}, P_{3}, P_{5}\right\}$, respectively, does not contain $P_{3}$. Let $H_{1}, H_{2}$ be two hyperplanes, respectively, containing these planes. Note that $P_{4} \in K$ and there exist two of $\left\{K, H_{1}, H_{2}\right\}$ containing $P_{i}$ for any $1 \leq i \leq 6, i \neq 4$. By the definition of covering, we have $2 P_{1}+2 P_{2}+$ $2 P_{3}+P_{4}+2 P_{5}+2 P_{6}$ is $(3,2)$-covered.

Since $P_{1}+\cdots+P_{6}$ is $(2,2)$-covered and $2 P_{1}+\cdots+2 P_{6}$ is $(3,1)$-covered, we have $3 P_{1}+\cdots+3 P_{3}$ is $(5,2)$-covered. Thus to prove $3 P_{1}+\cdots+3 P_{3}$ is $(5,3)$-covered, we need only to check for $i=2$, which is straightforward.

From Proposition 3.5 and Corollary 3.6, we now consider covering properties of some particular sets of $n+2$ almost equimultiple fat points in $\mathbb{P}^{n}$ with an assumption that the number of points having multiplicities $k-1$ is not more than two. 
Proposition 3.7. With the hypothesis as in Proposition 3.5, for any integer $k \geq 2$, we have:

(1) $m_{1} P_{1}+k P_{2}+\cdots+k P_{n+1}+m_{n+2} P_{n+2}$, for $m_{1}, m_{n+2} \geq 1, m_{1}+m_{n+2}=2 k-1$, is $(s, k-1)$-covered where $s=3 k-3$ if $d=1$ and $s=2 k-1$ if $d \geq 2$;

(2) $k P_{1}+k P_{2}+\cdots+k P_{n+2}$ is $(s, k-1)$-covered, where $s=3 k-2$ if $d=1$ and $s=$ $2 k-1$ if $d \geq 2$;

(3) $k P_{1}+k P_{2}+\cdots+k P_{n+2}$ is ( $\left.s, k\right)$-covered, where $s=3 k-1$ if $d=1, s=2 k-1$ if $d \geq 3, n+k \geq 7$ and $s=2 k$ otherwise;

(4) $\quad(k-1) P_{1}+k P_{2}+\cdots+k P_{n+1}+(k-1) P_{n+2}$ is $(s, k-1)$-covered, where $s=3 k-$ 4 if $d=1, k \geq 3$ and $s=2 k-1$ if $d \geq 2$ or $k=2$.

Proof. Assume that $P_{i}=e_{i}, i=1, \ldots, n$. We prove mainly by induction on $k$.

(1) For $k=2$, the assertion is attained by Corollary 3.6. Suppose that the conclusion holds for $k \geq 2$. We know from Proposition 3.5 that $P_{1}+\cdots+P_{n+2}$ is $(3,2)$-covered if $d=1$ and it is $(2,2)$-covered if $d \geq 2$. This implies that $\left(m_{1}+1\right) P_{1}+(k+1) P_{2}+\cdots$ $+(k+1) P_{n+1}+\left(m_{n+2}+1\right) P_{n+2}$ is $(3 k, k)$-covered if $d=1$ and $(2 k+1, k)$-covered if $d \geq 2$. Thus the conclusion holds for $k+1$ and hence the proof is complete.

(2) For $k=2$, since $2 P_{1}+\cdots+2 P_{n+2}$ is $(4,1)$-covered if $d=1$ and it is $(3,1)$-covered if $d \geq 2$ by Proposition 3.5, the conclusion follows. The same argument as in case (1) works for this case.

(3) From the above, we have $k P_{1}+k P_{2}+\cdots+k P_{n+2}$ is $(3 k-2, k-1)$-covered if $d=1$ and $(2 k-1, k-1)$-covered if $d=2$. This implies that $k P_{1}+k P_{2}+\cdots+k P_{n+2}$ is $(3 k-1, k)$-covered if $d=1$ and $(2 k, k)$-covered if $d=2$.

For $d \geq 3$, from Proposition 3.5 , we see that $2 P_{1}+\cdots+2 P_{n+2}$ is $(3,2)$-covered if $n \geq 5$ and $3 P_{1}+\cdots+3 P_{6}$ is $(5,3)$-covered for $n=4$ by Corollary 3.6. Suppose that $k P_{1}+k P_{2}+\cdots+k P_{n+2}$ is $(2 k-1, k)$-covered if $n \geq 5, k \geq 2$ or $n=4, k \geq 3$. Since $P_{1}+$ $\cdots+P_{n+2}$ is $(2,2)$-covered, we have $(k+1) P_{1}+\cdots+(k+1) P_{n+2}$ is $(2 k+1, k+1)$ covered for $n \geq 5, k \geq 2$ or for $n=4, k \geq 3$. By the induction principle, we have $k P_{1}+$ $k P_{2}+\cdots+k P_{n+2}$ is $(2 k-1, k)$-covered for $d \geq 3$ and any $n+k \geq 7$. Finally, for the case $n=4, k=2$, we have $2 P_{1}+\cdots+2 P_{6}$ is $(3,1)$-covered, hence it is $(4,2)$-covered. This completes the proof of (3).

(4) From case (1), we see that $(k-1) P_{1}+k P_{2}+\cdots+k P_{n+1}+(k-1) P_{n+2}$ is $(2 k-$ $1, k-1)$-covered if $d \geq 2$. For $k=2$, we have $P_{1}+2 P_{2}+\cdots+2 P_{n+1}+P_{n+2}$ is $(3,1)$ covered by Corollary 3.6.

Now, consider the case $d=1, k \geq 3$. If $k=3$, we know from Corollary 3.6 that $2 P_{1}+3 P_{2}+\cdots+3 P_{n+1}+2 P_{n+2}$ is $(5,2)$-covered. Suppose that $(k-1) P_{1}+k P_{2}+$ $\cdots+k P_{n+1}+(k-1) P_{n+2}$ is $(3 k-4, k-1)$-covered. We see from Proposition 3.5 that $P_{1}+\cdots+P_{n+2}$ is $(3,2)$ covered. Therefore, $k P_{1}+(k+1) P_{2}+\cdots+(k+1) P_{n+1}+$ $k P_{n+2}$ is $(3 k-1, k)$-covered. This means that the conclusion holds for $k+1$, hence the proof is complete.

The above results yield the following covering property of a general set of $n+2$ almost equimultiple fat points in a relationship to the general Segre bound $T_{Z}$ and with an assumption that the point $P=e_{0}$ has multiplicity $k-1$.

Corollary 3.8. Let $X:=\left\{P_{1}, \ldots, P_{n+3}\right\}$ be a non-degenerate set of distinct points, not in general position in $\mathbb{P}^{n}, n \geq 4$, such that there are no $n+2$ points of $X$ lying on 
a hyperplane. Let $d$ be the smallest number such that there exists a $d$-plane containing $d+2$ points of $X$. Assume that $P_{n+3}=e_{0}$ and $P_{1}, \ldots, P_{d}, P_{n+2}, P_{n+3}$ lie on a d-plane. Let $k \geq 2$ be an integer, and let $Z:=m_{1} P_{1}+\cdots+m_{n+3} P_{n+3}$, where $k-1 \leq m_{i} \leq k$, $i=1, \ldots, n+2$ and $m_{n+3}=k-1$. Set

$$
T_{j}:=\max \left\{\left[\frac{1}{j}\left(m_{i_{1}}+m_{i_{2}}+\cdots+m_{i_{q}}+j-2\right)\right] \mid P_{i_{1}}, P_{i_{2}}, \ldots, P_{i_{q}} \text { lie on a } j \text {-plane }\right\}
$$

and

$$
T_{Z}:=\max \left\{T_{j} \mid j=1, \ldots, n\right\} .
$$

Then $m_{1} P_{1}+\cdots+m_{n+2} P_{n+2}$ is $\left(T_{Z}, k-1\right)$-covered.

Proof. As usual, we assume that $P_{i}=e_{i}, i=1, \ldots, n$. We divide the proof into three cases.

Case 1: there exist two points in $X$ with multiplicities $k$. If $m_{1}=m_{n+2}=k$, then $T_{Z}=3 k-2$ if $d=1$ and $T_{Z} \geq T_{1}=2 k-1$ if $d \geq 2$. From Proposition 3.7, we have $k P_{1}+\cdots+k P_{n+2}$ is $\left(T_{Z}, k-1\right)$-covered, hence $m_{1} P_{1}+\cdots+m_{n+2} P_{n+2}$ is $\left(T_{Z}, k-1\right)$-covered.

If $m_{1}+m_{n+2}=2 k-1$, then $T_{Z} \geq 3 k-3$ if $d=1$ and $T_{Z} \geq T_{1}=2 k-1$ if $d \geq 2$. From Proposition 3.7, we have $m_{1} P_{1}+k P_{2}+\cdots+k P_{n+1}+m_{n+2} P_{n+2}$ is $\left(T_{Z}, k-1\right)$-covered, hence $m_{1} P_{1}+\cdots+m_{n+2} P_{n+2}$ is $\left(T_{Z}, k-1\right)$-covered.

If $m_{1}=m_{n+2}=k-1$, then $T_{Z} \geq 3 k-4$ if $d=1, k \geq 3$ and $T_{Z} \geq T_{1}=2 k-1$ if $d \geq 2$ or $k=2$. From Proposition 3.7, we have $(k-1) P_{1}+k P_{2}+\cdots+k P_{n+1}+(k-1) P_{n+2}$ is $\left(T_{Z}, k-1\right)$-covered, hence $m_{1} P_{1}+\cdots+m_{n+2} P_{n+2}$ is $\left(T_{Z}, k-1\right)$-covered.

Case 2: there exists only one point in $X$ with multiplicity $k$. Let $1 \leq t \leq n+2$ such that $m_{t}=k$.

If $d=1$ and $t \in\{1, n+2\}$, it means that $m_{1}+m_{n+2}=2 k-1$, then $T_{Z}=3 k-3$. By Proposition 3.7, $m_{1} P_{1}+k P_{2}+\cdots+k P_{n+1}+m_{n+2} P_{n+2}$ is $\left(T_{Z}, k-1\right)$-covered, hence $m_{1} P_{1}+\cdots+m_{n+2} P_{n+2}$ is $\left(T_{Z}, k-1\right)$-covered.

If $d=1$ and $1<t<n+2$, then $T_{Z} \geq 3 k-4$. By Proposition 3.7, $(k-1) P_{1}+k P_{2}+$ $\cdots+k P_{n+1}+(k-1) P_{n+2}$ is $(3 k-4, k-1)$-covered for $k \geq 3$. For $k=2$, we see from Proposition 3.5 that $P_{1}+\cdots+P_{t-1}+2 P_{t}+P_{t+1}+\cdots+P_{n+2}$ is $(2,1)$-covered. Thus $m_{1} P_{1}+\cdots+m_{n+2} P_{n+2}$ is $\left(T_{Z}, k-1\right)$-covered.

If $d \geq 2$, then $T_{Z}=T_{1}=2 k-2$. We will prove that

$$
(k-1) P_{1}+\cdots+(k-1) P_{t-1}+k P_{t}+(k-1) P_{t+1}+\cdots+(k-1) P_{n+2}
$$

is $(2 k-2, k-1)$-covered by induction on $k$. If $k=2$, it follows from Proposition 3.5 that $P_{1}+\cdots+P_{t-1}+2 P_{t}+P_{t+1}+\cdots+P_{n+2}$ is $(2,1)$-covered. The conclusion holds for $k=2$. Suppose that the conclusion holds for $k$. We know that $P_{1}+\cdots+P_{n+2}$ is $(2,2)$ covered for $d \geq 2$. This means that $k P_{1}+\cdots+k P_{t-1}+(k+1) P_{t}+k P_{t+1}+\cdots+k P_{n+2}$ is $(2 k, k)$-covered for $d \geq 2$. The conclusion holds for $k+1$.

Case 3: all points in $X$ have multiplicities $k-1$. For $k=2$, we have $T_{Z}=T_{n}=2$ and $P_{1}+\cdots+P_{n+1}$ is $(2,1)$-covered. The conclusion holds for $k=2$. If $k \geq 3$, then $T_{Z}=$ $3(k-1)-1$ if $d=1, T_{Z}=2(k-1)-1$ for $d \geq 3, n+k-1 \geq 7$ and $T_{Z}=2(k-1)$ otherwise. Proposition 3.7 now shows that $(k-1) P_{1}+\cdots+(k-1) P_{n-1}$ is $\left(T_{Z}, k-1\right)$ covered. 


\subsection{Regularity index of a set of $n+3$ almost equimultiple fat points}

We now check the conjecture for any non-degenerate set of $n+3$ almost equimultiple fat points in $\mathbb{P}^{n}$.

Proposition 3.9. Let $X:=\left\{P_{1}, \ldots, P_{n+3}\right\}$ be a non-degenerate set of distinct points, not in general position in $\mathbb{P}^{n}, n \geq 4$, such that there are no $n+2$ points of $X$ lying on a hyperplane. Let $k \geq 2$ be an integer and let $Z:=m_{1} P_{1}+\cdots+m_{n+3} P_{n+3}$, where $k-1 \leq$ $m_{i} \leq k, i=1, \ldots, n+3$. Set

$$
T_{j}:=\max \left\{\left[\frac{1}{j}\left(m_{i_{1}}+m_{i_{2}}+\cdots+m_{i_{q}}+j-2\right)\right] \mid P_{i_{1}}, P_{i_{2}}, \ldots, P_{i_{q}} \text { lie on a } j \text {-plane }\right\}
$$

and

$$
T_{Z}:=\max \left\{T_{j} \mid j=1, \ldots, n\right\} .
$$

Then there exists a point $P_{i_{0}} \in X$ such that if $P_{i_{0}}=e_{0}$, we have $\sum_{j \neq i_{0}} m_{j} P_{j}$ is $\left(T_{Z}, m_{i_{0}}\right)$ covered. Consequently,

$$
\operatorname{reg}\left(R /\left(J+\wp_{i_{0}}^{m_{i_{0}}}\right)\right) \leq T_{Z},
$$

where $\wp_{j}$ is the ideal corresponding to $P_{j}$ and $J:=\bigcap_{j \neq i_{0}} \wp_{j}^{m_{j}}$.

Proof. Let $d$ be the smallest number such that there exists a $d$-plane containing $d+2$ points of $X$. The proof will be divided into two cases.

Case 1: there exists a d-plane $K$ containing $d+2$ points of $X$, one of which has multiplicity $k-1$. Choose $P_{i_{0}} \in K$, a point of $X$ with multiplicity $k-1$ and assume that $P_{i_{0}}=e_{0}$. Then Proposition 3.7 shows that $\sum_{j \neq i_{0}} m_{j} P_{j}$ is $\left(T_{Z}, m_{i_{0}}\right)$-covered.

Case 2: all points in any $d$-plane containing $d+2$ points of $X$ have multiplicities $k$. Consider the case $d \leq 2$ or $n+k \geq 7$, then $T_{Z}=3 k-1$ if $d=1, T_{Z}=2 k-1$ if $d \geq 3$ and $T_{Z}=2 k$ otherwise. Choose $P_{i_{0}}$ to be a point in a $d$-plane containing $d+2$ points of $X$ and assume that $P_{i_{0}}=e_{0}$. Proposition 3.7 shows that $k P_{1}+\cdots+k P_{n+2}$ is $\left(T_{Z}, k\right)$-covered. This implies that $\sum_{j \neq i_{0}} m_{j} P_{j}$ is $\left(T_{Z}, m_{i_{0}}\right)$-covered.

Consider the case $n=4, k=2$ and $d=3$. Choose $P_{i_{0}}=P_{7}$ to be a point on a 3-plane containing $P_{1}, P_{2}, P_{3}, P_{6}, P_{7}$ with multiplicities 2 .

If $m_{4}=m_{5}=2$ then $T_{Z}=T_{4}=4$. Since $2 P_{1}+\cdots+2 P_{6}$ is $(3,1)$-covered by Proposition 3.5 , it is $(4,2)$-covered.

If $2 \leq m_{4}+m_{5} \leq 3$ then $T_{Z}=3$. We can assume that $m_{4}=1$. We know from Corollary 3.6 that $2 P_{1}+2 P_{2}+2 P_{3}+P_{4}+2 P_{5}+2 P_{6}$ is $(3,2)$-covered. This implies that $2 P_{1}+2 P_{2}+2 P_{3}+m_{4} P_{4}+m_{5} P_{5}+2 P_{6}$ is $(3,2)$-covered.

The proof is complete.

TheOREM 3.10. Let $n, k$ be positive integers and let $k \geq 2$. Let $X:=\left\{P_{1}, \ldots, P_{n+3}\right\}$ be a set of non-degenerate distinct points in $\mathbb{P}^{n}$ and let $Z:=m_{1} P_{1}+\cdots+m_{n+3} P_{n+3}$, where $k-1 \leq m_{i} \leq k, i=1, \ldots, n+3$. Let $\wp_{j}$ be the ideal corresponding to $P_{j}$ and let $I:=$ $\wp_{1}^{m_{1}} \cap \cdots \cap \wp_{n+3}^{m_{n+3}}$. Then

$$
\operatorname{reg}(R / I) \leq T_{Z},
$$

where

$$
T_{j}:=\max \left\{\left[\frac{1}{j}\left(m_{i_{1}}+m_{i_{2}}+\cdots+m_{i_{q}}+j-2\right)\right] \mid P_{i_{1}}, P_{i_{2}}, \ldots, P_{i_{q}} \text { lie on a } j \text {-plane }\right\}
$$


and

$$
T_{Z}:=\max \left\{T_{j} \mid j=1, \ldots, n\right\} .
$$

Proof. We only need to consider the case where $X$ is non-general position and $n \geq 4$. Other cases follow from $[4,7,11]$.

Suppose that there exists a hyperplane containing $n+2$ points, say $P_{1}, \ldots, P_{n+2}$ of $X$. Assume that $P_{n+3}=e_{0}$ and set $Y:=\sum_{j=1}^{n+2} m_{j} P_{j}, J:=\bigcap_{j=1}^{n+2} \wp_{j}^{m_{j}}$. Then we can consider that $P_{1}, \ldots, P_{n+2}$ are contained in a projective space $\mathbb{P}^{n-1}$, and let $Y^{\prime}$ be the set of fat points in $\mathbb{P}^{n-1}$ corresponding to $Y$. By induction, we have reg $\left(Y^{\prime}\right) \leq T_{Y^{\prime}}$. By Lemma 2.4, we have $\operatorname{reg}(R / J)=\operatorname{reg}(Y) \leq T_{Y^{\prime}} \leq T_{Y} \leq T_{Z}$. Moreover, Corollary 2.3 implies

$$
\operatorname{reg}\left(R /\left(J+\wp_{n+3}^{m_{n+3}}\right)\right) \leq T_{Z} .
$$

By Lemma 2.1, we have

$$
\operatorname{reg}(R / I)=\max \left\{m_{n+3}-1, \operatorname{reg}(R / J), \operatorname{reg}\left(R /\left(J+\wp_{n+3}^{m_{n+3}}\right)\right)\right\} \leq T_{Z} .
$$

We now consider the case that there is no hyperplane containing $n+2$ points of $X$. By Proposition 3.9, there exists $P_{i_{0}} \in X$ such that

$$
\operatorname{reg}\left(R / J+\wp_{i_{0}}^{m_{i_{0}}}\right) \leq T_{Z}
$$

where $J:=\bigcap_{j \neq i_{0}} \wp_{j}^{m_{j}}$.

Moreover, the ideal $J$ corresponds to $n+2$ non-degenerate points in $\mathbb{P}^{n}$ and we have, by [1], that

$$
\operatorname{reg}(R / J) \leq T_{Y} \leq T_{Z}
$$

where $Y:=\sum_{j \neq i_{0}} m_{j} P_{j}$.

By Lemma 2.1, we have

$$
\operatorname{reg}(R / I)=\max \left\{m_{i_{0}}-1, \operatorname{reg}(R / J), \operatorname{reg}\left(R /\left(J+\wp_{i_{0}}^{m_{i_{0}}}\right)\right)\right\} \leq T_{Z} .
$$

The proof is complete.

Acknowledgements. The authors are grateful to the referees for pointing out mistakes in the manuscript and for many precious comments which are extremely useful for strengthening the results of the paper. The authors are supported by the Vietnam National Foundation for Science and Technology Development (NAFOSTED).

\section{REFERENCES}

[1] B. Benedetti, G. Fatabbi and A. Lorenzini. Segre's bound and the case of $n+2$ fat points of $\mathbb{P}^{n}$. Comm. Algebra 40 (2012), 395-403.

[2] M. V. Catalisano. Linear systems of plane curves through fixed fat points of $\mathbb{P}^{2}$. J. Algebra 142 (1991), 81-100.

[3] M. V. Catalisano. Fat points on a conic. Comm. Algebra 19 (1991), 2153-2168.

[4] M. V. Catalisano, N. V. Trung and G. Valla. A sharp bound for the regularity index of fat points in general position. Proc. Amer. Math. Soc. 118 (1993), 717-724.

[5] E. D. Davis and A. V. Geramita. The Hilbert function of a special class of 1-dimensional Cohen-Macaulay graded algebras. The Curves Seminar at Queen's (Queen's Papers in Pure and Applied Mathematic, 67). Queen's University, Kingston, Ontario, 1984, pp. 1-29. 
[6] G. Fatabbi. Regularity index of fat points in the projective plane. J. Algebra 170 (1994), 916-928.

[7] G. Fatabbi and A. Lorenzini. On a sharp bound for the regularity index of any set of fat points. J. Pure Appl. Algebra 161 (2001), 91-111.

[8] W. Fulton. Algebraic Curves (Mathematics Lecture Note Series). Benjamin, New York, 1969.

[9] A. Ooishi. Castelnuovo's regularity of graded rings and modules. Hiroshima Math. J. 12 (1982), 627-649.

[10] B. Segre. Alcune questioni su insiemi finiti di punti in Geometria Algebrica. Atti del Convegno Internazionale di Geometria Algebrica (Universitá di Torino, 1961). Tipi L. Rattero, Torino, 1962, 15-33.

[11] P. V. Thien. On Segre bound for the regularity index of fat points in $\mathbb{P}^{2}$. Acta Math. Vietnam. 24 (1999), 75-81.

[12] P. V. Thien. Segre bound for the regularity index of fat points in $\mathbb{P}^{3}$. J. Pure Appl. Algebra 151 (2000), 197-214.

[13] P. V. Thien. Sharp upper bound for the regularity of zero-schemes of double points in $\mathbb{P}^{4}$. Comm. Algebra 12 (2002), 5825-5847.

[14] N. V. Trung. An algebraic approach to the regularity index of fat points in $\mathbb{P}^{n}$. Kodai Math. J. 17 (1994), 382-389.

\title{
Nguyen Chanh Tu
}

Center of Advanced Programs

Danang University of Technology

Danang

Vietnam

(E-mail: nctu@dut.udn.vn;nctu2000@yahoo.com)

\author{
Tran Manh Hung \\ Department of Mathematics \\ Quangbinh University \\ Quangbin \\ Vietnam
}

(E-mail:tmhung2007@gmail.com) 